\title{
FUNCTIONAL ANALYSIS OF G12E MUTATION OF AVPR2 GENE IN TURKISH PATIENTS WITH DIABETES INSIPIDUS
}

\author{
Beril Erdem¹, Emel Saglar1, Tugce Karaduman ${ }^{1}$, Merve Ozcan ${ }^{1}$, Ferhat Deniz², Hatice Mergen ${ }^{1}$ \\ 1 Department of Biology, Faculty of Science, Hacettepe University, Beytepe, Ankara, 06800, Turkey \\ 2 GATA Haydarpasa Teaching Hospital, Department of Endocrinology and Metabolism, Istanbul, 34668, Turkey
}

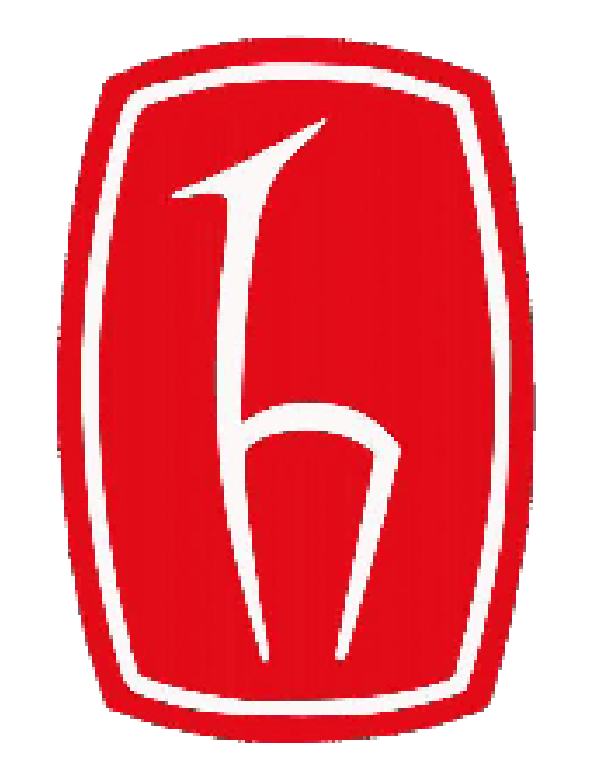

\section{OBJECTIVES}

Diabetes insipidus (DI) is a rare disorder which is characterized with inability to concentrate urine because of severe liquid-balance impairment. Mutations in AVPR2 gene is one of the causes of DI. AVPR2 is a $\mathrm{G}$ protein coupled receptor (GPCR) and its specific agonist is arginine vasopressin (AVP). When AVP binds to the AVPR2, which locates on the basolateral side of collecting duct principal cells of the kidney, it triggers accumulation of cAMP in the cell as a seconder messenger. If AVPR2 has a mutation, receptor could loose its function which is important for liquid balance of the body. The aim of this study is making functional characterization of G12E mutation of AVPR2 gene.

\section{METHODS}

G12E mutation was seen in three cases in our study. Functional characterization of this mutant is important for the therapeutic studies. G12E mutation was generated by site-directed mutagenesis strategy. For this purpose, pLV2R (a mammalian expression vector, from Dr. Angela Schulz, Leipzig University) was used and the mutant construct was checked with DNA sequencing. COS-7 cells were transfected with wild type and mutant construct and 72 hours after transfection, totally expression in the cell and cell surface expression of the mutant receptor was analyzed with total ELISA and cell surface ELISA experiments, respectively. In addition to this, cAMP accumulation assay has been performed after stimulation of mutant receptor with different concentrations of AVP. This study was supported by TUBITAK SBAG (Project numbers: 112S513 and 115S499).

\section{RESULTS}

G12E mutation showed reduced expression in total and also on cell surface. According to the ELISA results, mutant receptor showed $84.78 \% \pm 6.3(\mathrm{n}=7)$ total expression and $58.75 \% \pm 7.8(\mathrm{n}=8)$ cell surface expression compared to the wild type receptor. ELISA results can be seen in Figure 1. The results of cAMP accumulation assay are preliminary results and supported our ELISA results (were not shown).

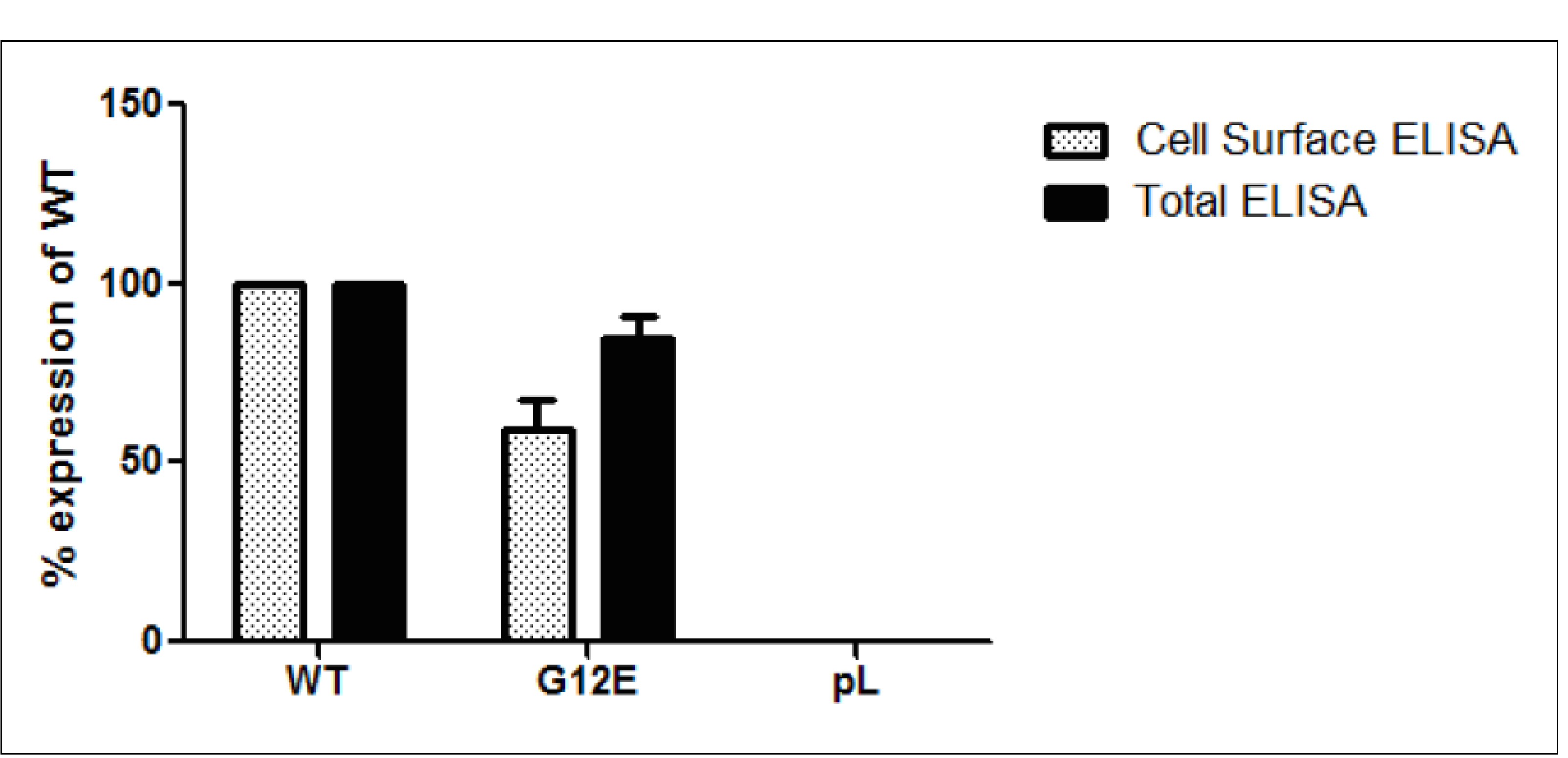

Figure 1. ELISA results of G12E mutant receptor, WT: wild type, pL: vector without $\mathrm{V} 2 \mathrm{R}$ sequence

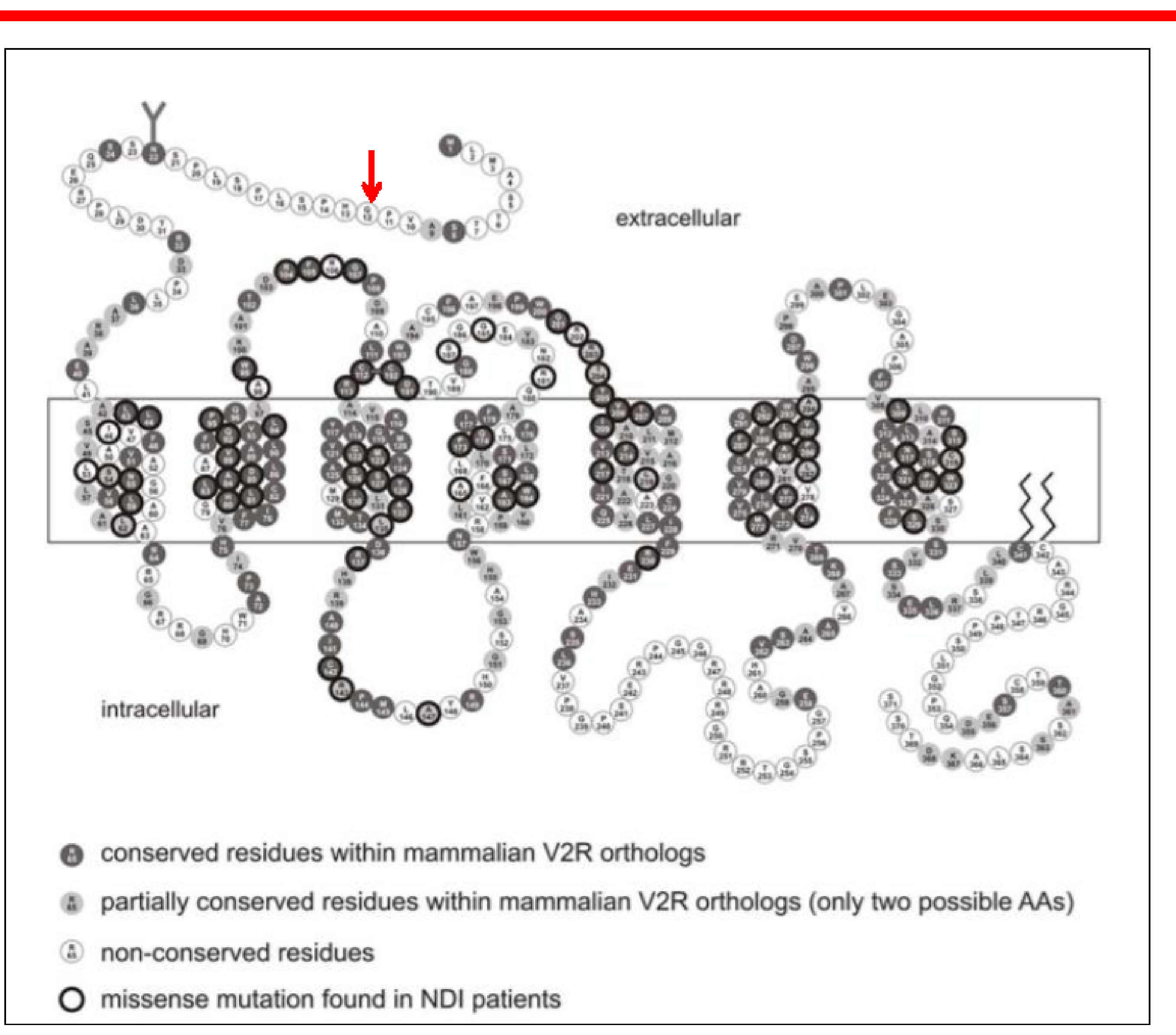

Figure 2. AVPR2 protein. G12E mutant was shown with red arrow (This picture were used from Boselt et al., 2009).

\section{CONCLUSIONS}

In conclusion, in vitro studies revealed that G12E mutation yields partial expression in the cell surface. At codon 12 , a change from glycine to glutamic acid may affect the cell surface expression of receptor because glycine is a small amino acid and it can fit both hydrophilic and hydrofobic environments. However, glutamic acid is a negatively charged amino acid and also it has a bigger side chain than glycine. We can say that changing in amino acid properties may cause both reduced total and cell surface expression of the mutant receptor in the cell. Supports of cAMP accumulation assay results which are preliminary showed that functional characterization of this mutation is important for understarnding the disease and shed light on treatments. For further assays to characterize mutant receptor, in silico studies and fluorescence imgaing of the cells that have mutant receptor will be done.

\section{References}

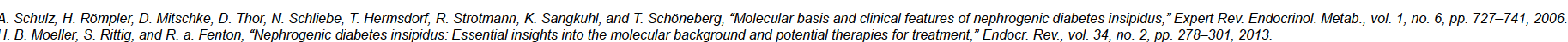
Wesche, P. . . T. Deen, and N. V. a M. Knoers, "Congenital nephrogenic diabetes insipidus: The current state of affairs," Pediatr. Nephrol, vol. 27, no. 12, pp. 2183-2204, 2012. 